\title{
Kernos
}

Revue internationale et pluridisciplinaire de religion grecque antique

$26 \mid 2013$

Varia

\section{Sandrine Dubel, Alain Montandon (éd.), Mythes sacrificiels et ragoûts d'enfants}

\section{Sergio Ribichini}

\section{QpenEdition \\ Journals}

Édition électronique

URL : http://journals.openedition.org/kernos/2155

DOI : 10.4000/kernos.2155

ISSN : 2034-7871

Éditeur

Centre international d'étude de la religion grecque antique

Édition imprimée

Date de publication : 10 octobre 2013

Pagination : 402-406

ISSN : 0776-3824

Référence électronique

Sergio Ribichini, «Sandrine Dubel, Alain Montandon (éd.). Mythes sacrificiels et ragoûts d'enfants », Kernos [En ligne], 26 | 2013, mis en ligne le 10 octobre 2013, consulté le 02 mars 2021. URL : http:// journals.openedition.org/kernos/2155; DOI : https://doi.org/10.4000/kernos.2155

Ce document a été généré automatiquement le 2 mars 2021.

Kernos 


\title{
Sandrine Dubel, Alain Montandon (éd.), Mythes sacrificiels et ragoûts d'enfants
}

\author{
Sergio Ribichini
}

\section{RÉFÉRENCE}

Sandrine Dubel, Alain Montandon (éd.), Mythes sacrificiels et ragoûts d'enfants, ClermondFerrand, Presses Universitaires Blaise Pascal, 2012. 1 vol. $14 \times 22 \mathrm{~cm}, 494$ p.

(Mythographies et sociétés). ISBN : 978-2-84516-519-9.

Enfants cuits et consommés : ce n'est pas un met qui flatte aisément le palais, ni des hommes, ni des dieux. Et les mythes grecs sont là pour l'établir en de riches nuances, pour le décliner en de nombreuses variations, pour le redire dans plusieurs récits. En premier lieu, l'avalement de ses enfants par Cronos, au début du monde, se referme sur l'obligation de les régurgiter, ce qui conduit à la fin de son pouvoir. Tué par son père Tantale et servi en ragoût aux dieux, lors d'un banquet des temps primitifs, Pélops est recomposé et gagne le trône, tandis que le coupable est condamné à souffrir d'un triple supplice éternel. Atrée et Thyeste, fils jumeaux de Pélops, s'opposent dans un registre similaire; le premier met en pièces les trois enfants de son frère, les fait bouillir et les sert à leur père; c'est Égisthe, né de l'inceste de Thyeste et sa fille Pélopia, qui finit par tuer Atrée et par rendre le royaume à son père. Zeus renverse la table que l'Arcadien Lycaon lui a apprêtée avec la chair d'un enfant (son propre fils Nyctimos, ou son petitfils Arcas) et foudroie Lycaon lui-même, qui selon d'autres récits est plutôt changé en loup. Les femmes acharnées qui s'agitent autour du chaudron où bouillit la chair de leurs enfants pour être servie à la table des hommes sont changées en oiseaux, aussi bien que leurs hommes cannibales : c'est ce qui arrive à Polytechnos transformé en picvert, à Aédon et Procné métamorphosées en rossignol, à Chélidon et Philomèle changées en hirondelle, à Térée en huppe et à Harpalycé en chalcis, un oiseau de proie. 
Crime alimentaire, le repas tecnophage des pères situé dans un contexte de luttes dynastiques et familiales révèle la barbarie des protagonistes et annonce leur glissement vers l'animalité; préparer et manger de la chair humaine c'est un comportement «bestial ». Les dieux, d'autre part, reconnaissent la viande qui leur est servie par les hommes lors de banquets qui sont une perversion du sacrifice d'animaux. Depuis toujours, les Olympiens s'indignent d'un tel repas et le refusent nettement: dans le monde régi par l'ordre de Zeus, il n'y a plus de place pour les coutumes dépassées du seigneur Cronos, qui ingurgitait consciemment ses enfants divins. Ce roi d'antan ne peut plus que régner chez les barbares, là où, peut-être, on honore encore les dieux avec des victimes humaines. Abandon du pouvoir, perte de la nature humaine, frontières de la civilisation : pour les Grecs qui racontaient ces mythes, c'est le modèle prométhéen du sacrifice animal qui domine les rapports hommes-dieux; et sur la terre, l'enfant mis en morceaux à l'époque du mythe, cuisiné et préparé pour son père, protège les bonnes règles conjugales et défend les relations de parenté les plus convenables. Commensalité et cannibalisme chez les Grecs, et pas seulement: au Proche Orient préclassique le récit du sacrifice manqué d'Isaac assurait le refus de cette coutume rituelle chez le peuple de Yahweh et répétait, avec l'immolation d'une victime animale de substitution, la juste formulation du «sacrifice" à Dieu. De même que le faisait, dans les mythes hellènes, la biche qui avait pris la place d'Iphigénie, fille d'Agamemnon, sur l'autel d'Artémis.

Ce sont des questions qui ont longtemps attiré l'attention des classicisants et des historiens des religions, et qui se prêtent encore largement à l'analyse. Des travaux comme Homo necans de Walter Burkert (1972), Lykaon de Giulia Piccaluga (1968) et Cannibalisme et immortalité de Monique Halm Tisserant (1993) restent à mon avis des étapes fondamentales de l'historiographie sur l'anthropophagie comme marqueur d'identité, signe d'exclusion, élément fondateur d'idéologies, d'usages et de rites. Mais de nombreuses nuances et des enjeux esthétiques, éthiques, sociaux et religieux sont encore à explorer, et le livre que l'on présente ici dévoile combien des éléments de réflexion peuvent encore émaner de l'analyse de ces récits, tant dans leurs contextes spécifiques que dans leurs redéploiements en des temps et des lieux même très éloignés de l'antiquité classique et du Proche-Orient ancien. Sandrine Dubel et Alain Montandon ont ici réuni les résultats de recherches préalablement présentées à l'occasion d'un colloque organisé à l'Université Blaise Pascal en octobre 2008 autour des violences commises par les parents sur les enfants : de l'exposition du nouveau-né au sacrifice de la descendance, de l'avalement d'enfants par les dieux à leur consommation par les ogres et surtout par les pères. Très habilement, les éditeurs ont amalgamés les diverses études en les distribuant en trois axes d'analyse: (1) «Archétypes antiques et réécritures: les scandales du ventre", avec dix contributions; (2) "Histoire de la bouche : de la chair du conte à l'origine du livre", avec six essais; et (3) «Pratiques, fantasmes et idéologies : vers une rhétorique de l'infanticide ", avec neuf articles.

Toute la première partie considère la thématique de l'infanticide et des acteurs coupables de la transgression dans des textes antiques et dans leurs réécritures modernes, de la Bible à Goethe. Marie-Christine Gomez-Géraud ouvre la section sur l'épisode du sacrifice d'Abraham et relit le texte original de Genèse 22 à la lumière de ses représentations dans l'art et la littérature de l'Occident, pour comprendre la pertinence ponctuelle des dénomination de "sacrifice " et de "rite de substitution", pour une scène qui met en jeu des éléments symboliques complexes. Aux origines du monde se pose l'étude de Christine Hunzinger qui s'occupe d'une manière comparative du motif 
de l'enfant avalé chez Hésiode et dans les antécédents orientaux de sa Théogonie, par exemple dans le mythe hourro-hittite de Kumarbi et le poème babylonien de la création; le récit hésiodique révèle à son avis la cohérence d'un réseau d'images multiples autour du ventre comme endroit paradoxal, conçu comme lieu de gestation de l'être, espace de rétention et cavité susceptible de manipulation rusée. L'engloutissement des enfants de Cronos dans la Théogonie est l'objet aussi de l'étude de Charles Delattre. Pour lui, l'avalement, l'étouffement et la régurgitation représentent une triade de symptômes que l'on retrouve même dans des textes physiologiques et médicaux grecs sur le statut de l'enfant et la nature du fotus, l'avortement et l'accouchement. Anne Gangloff s'engage à relire l'histoire de Pélops, en analysant la manière dont les Anciens l'ont reçue et réinterprétée depuis le début du $\mathrm{V}^{\mathrm{e}} \mathrm{s}$. av. J.-C. jusqu'au $\mathrm{V}^{\mathrm{e}} \mathrm{s}$. de notre ère; elle montre comment ce mythea acquis très tôt une dimension politique qui sublimait en quelque sorte l'infanticide. À partir de l'époque hellénistique, Pélops est en effet devenu le prototype du roi sacré protégé par les dieux, modèle qui s'est historiquement développé après Alexandre le Grand. Vient ensuite l'étude de Christine Kossaifi qui s'occupe du sacrifice que Lycaon fit à Zeus de son petitfils Arcas, et des symbolismes qui entremêlent le loup, l'ours et le bouc aux origines mythiques de l'Arcadie; le sacrifice monstrueux prend ici la dimension d'un mythe ethnologique, tout en dessinant au cœur de l'antique Arcadie l'image poétique de l'homme primitif. L'histoire d'Itys, tué par sa mère et mangé par son père, est l'objet de l'analyse de Ludi Chazalon: l'iconographie des vases attiques du Ve s. av. J.-C. semble mettre l'accent sur l'innocence de la victime, l'extinction d'une lignée, la culpabilité des femmes, tandis que les crimes de l'homme sont à peine évoqués, presque soustraits aux regards; le meurtre et la cuisine de l'enfant sont mis en scène pour montrer la monstruosité absolue de la mère qui inverse le cours du monde en tuant son enfant et en l'abolissant dans le père. Cet infanticide retient aussi l'attention d'Hélène Vial qui prend comme point de départ la version offerte par les Métamorphoses d'Ovide, pour définir la transformation en oiseau que le poète traite comme une mise en œuvre de la tension, parfois épanouissante mais le plus souvent destructrice, entre identité et altérité. Sabine Gruffat s'intéresse au traitement du sacrifice d'Iphigénie chez Rotrou et Racine : au XVII ${ }^{\mathrm{e}}$., l'histoire de cette héroïne est conçue comme un exemplum païen susceptible d'édifier un public chrétien par un sublime moral et religieux. Sylvie Humbert-Mougin examine de son côté le succès connu au siècle suivant par le festin de Thyeste et les enjeux de sa réactivation dans les ouvrages de Crébillon, Voltaire et Foscolo, aussi bien que les stratégies déployées par ces trois dramaturges. En partant de l'Iphigénie d'Euripide, Markus Winkler examine les adaptations du mythe grec au cœur des valeurs humanistes exprimées par Goethe dans la ballade de la Fiancée de Corinthe (Die Braut von Corinth, 1797).

Le deuxième axe porte sur l'enfant sacrifié, dévoré ou exposé, tant dans les contes traditionnels d'Occident et d'Afrique que dans des récits littéraires et romans contemporains, qui réinterprètent les scénarios mythiques de l'enfant menacé d'avalement pour raconter des histoires organisées autour de la puissance de la parole de la victime et nous mener aux mécanismes de la création littéraire. Daniel Aranda discute du récit "Ma mère m'a tué, mon père m'a mangé » (numéro 720 selon la classification des contes folkloriques de Aarne-Thompson, 1928), dans lequel infanticide culinaire et tecnophagie prennent sens dans une petite guerre familiale qui transporte le public au seuil du sacré tel qu'il était exprimé déjà dans le mythe d'Itys. Martine Courtois questionne les versions européennes, nord-africaines et africaines du 
conte-type 327 sur l'ogre qui mange des ragoûts d'enfants et qui finit par se faire duper par la parole rusée du héros enfantin. Lorine Bost retrouve cette sorte d'inversion, de la polarité ingestive de la bouche en une polarité formulative, dans deux réécritures romanesques contemporaines du Poucet, Le Tambour de Günter Grass (1959) et Magnus de Sylvie Germain (2005), qu'elle étudie à partir des mythes de Cronos et de Procné réinterprétés par la tradition chrétienne. Alain Montandon analyse le conte Ignaz Denner d'E.T.A. Hoffmann (1814) qui narre l'histoire d'un brigand qui boit le sang de ses enfants pour garder une éternelle jeunesse et discute la figure du père vampirique qu'inverse le processus de la naissance. Éric Lysøe étudie d'autres textes des XIX ${ }^{e}$ et $\mathrm{XX}^{\mathrm{e}}$ s. dans lesquels l'enfant apparaît comme monstre et bourreau plutôt que comme victime, tandis que Liana Nissim analyse quelques œuvres de la production littéraire africaine francophone actuelle, mettant en scène des enfants victimes sacrificielles, pour discuter de la destructuration de systèmes des valeurs et de la dissolution des croyances traditionnelles résultant de l'entrée de l'Afrique dans la modernité.

Alors que les deux premiers axes offrent des approches philologiques des sources textuelles et iconographiques, les contributions de la troisième partie du volume s'intéressent plutôt aux réinvestissements des motifs et des figures mythiques de l'enfant tué dans d'autres types de discours. Les auteurs explorent ici les stratégies de la mise en scène des infanticides et d'autres horreurs culinaires, entre vérité et fiction, dans les littératures de l'Antiquité et des temps modernes. Giuseppina Paola Viscardi revient sur le sacrifice d'Iphigénie à Aulis et sur la figure d'Artémis taurique, destinataire de ce crime rituel, pour examiner la façon dont le mythe pouvait s'entremêler aux rituels initiatiques pratiqués en l'honneur de la déesse à Brauron, à Mounychie et à Athènes. Anne de Cremoux étudie le mythe et sa représentation sur la scène tragique, discutant du sacrifice volontaire du jeune Ménécée dans les Phéniciennes d'Euripide. Aurélie Damet met en évidence les raisons amenant à l'exposition des enfants dans la cité athénienne à l'époque classique, le plus souvent l'abandon de petites filles. Stavroula Kefallonitis examine la façon dont le motif de la violence infanticide informe le discours du pouvoir politique, propre ou métaphore, dans l'histoire semi-mythique de Périandre de Corinthe et dans le portrait du tyran Aristodème de Cumes. C'est au poème 64 de Catulle, relatant les noces de l'immortelle Thétis et du mortel Pélée, que s'intéresse Jean-Pierre De Giorgio, pour remarquer dans son épilogue brutal les modalités romaines de décliner les crimes impies de l'époque des origines racontés par les mythes grecs. Stéphanie Wyler étudie les prétendues ragoûts d'enfants dans les orgies dionysiaques, de la construction du mythe orphique sur le dieu démembré, bouilli, rôti et dévoré par les Titans, au compte-rendu des bacchanales de Tite-Live. Les agapes nocturnes tecnophages dont on accusait les premières communautés chrétiennes et leurs représentations ironiques chez les apologistes sont l'objet de l'étude de Stéphane Solier qui met en évidence l'utilisation de détails et d'un vocabulaire directement empruntés à la tradition mythique classique pour retourner ces crimes contre leurs détracteurs. Viennent enfin la réflexion de Mathilde Bernard, sur l'élaboration littéraire de la figure de la mère cannibale lors des dangers (tels que le siège de Jérusalem en 70 apr. J.-C.), révisée dans de différents contextes narratifs, et celle de Nicolas Cremona sur les infanticides classiques (Médée et Atrée, en particulier) revisités dans les histoires tragiques de la Renaissance et à l'âge baroque : de l'histoire événementielle au discours, du mythe à la fiction littéraire.

On pourra remarquer que quelques-unes des histoires examinées ne partagent pas pleinement le thème de l'enfant "mis en ragoût", et que certains contributeurs 
utilisent de manière impropre des termes qui ne sont pas exactement des synonymes (mythe, conte, légende, récit, tradition). On notera également qu'il faut toujours historiciser des notions comme " sacrifice ", " infanticide ", " mise à mort rituelle", pour pouvoir correctement placer dans chaque contexte le motif et la façon de tuer une victime humaine avec ou sans destinataire (voir Angelo Brelich, Presupposti del sacrificio umano, a cura di Andrea Alessandri, Rome: Editori Riuniti, 2011). Mais au fond, on reconnaîtra facilement que le livre atteint l'objectif déclaré par les organisateurs, chaque contributeur s'étant interrogé sur les infanticides commis par les parents dans les formes les plus transgressives et dans différents domaines disciplinaires. On voit aussi dans le volume l'écho des recherches sur les violences intergénérationnelles menées à Clermont-Ferrand dans le cadre des programmes d'enquête du Centre d'Études sur les Littératures et la Sociopoétique de l'Université Blaise Pascal; et il est facile d'imaginer qu'un deuxième volume, consacré à la rébellion des enfants contre leurs parents et annoncé dans "l'Avant-goût", donnera lieu à d'autres réflexions fructueuses sur les représentations collectives des liens familiaux. Certainement, on y prendra en compte quelques travaux récents qui pourront être comparés avec ce livre. Ainsi, Àgnès A. Nagy a publié, en 2009, Qui a peur du cannibale? Récits antiques d'anthropophages aux frontières de l'humanité (Turnhout, Brepols), où elle développe soigneusement la thématique de la dévoration anthropophagique de l'antiquité grecque à l'empire romaine chrétien. En 2011, sous la direction de la même Àgnès A. Nagy et de Francesca Prescendi, plusieurs historiens des religions, anthropologues et archéologues ont étudié les rituels traditionnellement appelés "sacrifices humains ", dans leurs domaines respectifs de recherche, et discuté des problèmes méthodologiques relatifs : maintenant Sacrifices humains. Dossiers, discours, comparaisons (Turnhout, Brepols, 2013). Un autre ouvrage est sorti de presse la même année sur ce thème: P. Bonnechere, R. Gagné (éd.), Sacrifices humains. Perspectives croisées et représentations / Human sacrifice. Cross-cultural perspectives and representations (Liège, Presses Universitaires de Liège, 2013). Pour celui qui veut comprendre davantage comment, des premiers siècles de notre ère à l'époque contemporaine, s'est créée et transmise l'accusation d'homicide rituel imputée aux chrétiens et aux juifs, le livre de Marie-France Rouart, Le crime rituel ou le sang de l'autre (Paris, Berg International Éditeur, 1997) me paraît toujours d'une lecture profitable.

\section{AUTEURS}

\section{SERGIO RIBICHINI}

CNR - Roma 